VOL. $2(1970), 335-345$.

\title{
Some group laws equivalent to the commutative law
}

\author{
J. M. Gandhi
}

In this paper we prove the Theorem: A group satisfying the law (1) $[x, y]=[u, v, w]$, where $u, v, w$ are the entries from the set $\left\{x, x^{-1}, y, y^{-1}\right\}$ is always abelian. Earlier a few cases of this theorem were proved by Gupta. It is also proved that a group satisfying the law $[x, y]^{x^{2}}=[x, y, x]$ is always abelian.

\section{Introduction}

The purpose of the present paper is to prove the following:

THEOREM 1. A group $G$ satisfying the law

$$
[x, y]=[u, v, w]
$$

where $u, v$ and $w$ are entries from the set $\left\{x, x^{-1}, y, y^{-1}\right\}$, is always abelian.

Earlier a few cases of Theorem 1 were proved by Gupta [1].

We remark that using Theorem 1 , it can be proved that a group $G$ satisfying the law $[x, y]=[u, v, w, z]$ where $u, v, w, z$ are entries from the set $\left\{x, x^{-1}, y, y^{-1}\right\}$ implies that the group is abelian in certain cases. A few special cases were discussed by Gupta [1]. Because of the complexity we do not discuss this problem which the author has solved only partially.

Received 3 February 1970. 
Rejecting the cases when $u=v$ or $u=v^{-1}$ which trivially imply abelianness, we have 32 other possible combinations for (1.1). We shall group them into three different categories. The first category is when the right hand side of $(1.1)$ is of the form $\left[u, w, w^{-1}\right]$ or $\left[u, w, u^{-1}\right]$. There are sixteen possible cases for this category. The second category consists of eight expressions of the form $[x, y]=[u, w, x], u, w \neq x^{-1}$ or $[x, y]=[u, w, y], u, w \neq y^{-1}$. The remaining eight cases are to be discussed separately. They are:

$$
\begin{gathered}
{[x, y]=\left[x^{-1}, y, x^{-1}\right],\left[x, y^{-1}, y^{-1}\right],\left[x^{-1}, y^{-1}, x^{-1}\right],\left[y^{-1}, x^{-1}, y^{-1}\right],} \\
{\left[y^{-1}, x^{-1}, x^{-1}\right],\left[y, x^{-1}, x^{-1}\right],\left[x^{-1}, y^{-1}, y^{-1}\right] \text { and }\left[y^{-1}, x, y^{-1}\right] .}
\end{gathered}
$$

The first two cases were discussed by Gupta [1]. Separate proofs are given for the remaining cases. A few cases of categories 1 and 2 were also proved by Gupta but our general method will show how they can otherwise easily be proved.

We also prove

THEOREM 2. A group $G$ satisfying the zaw $[x, y]^{x^{2}}=[x, y, x]$ is always abelian.

\section{Notations and identities}

For two elements $x$ and $y$ in a group $G$, we define $x^{y}=y^{-1} x y$. The commutator of $x$ and $y$ is defined as

$$
[x, y]=x^{-1} y^{-1} x y
$$

and for $n>2$, a left normed commutator of weight $n$ is defined inductively as $\left[x_{1}, x_{2}, \ldots, x_{n}\right]=\left[\left[x_{1}, x_{2}, \ldots, x_{n-1}\right], x_{n}\right]$. We shall use the following identities which can be easily proved (see also Hall [2]).

$$
\begin{gathered}
{[x, y]=\left[x, y^{-1}\right]^{-y}=\left[x^{-1}, y\right]^{-x},} \\
{[x, y z]=[x, z][x, y]^{z},}
\end{gathered}
$$




$$
\left[u, w, w^{-1}\right]=[w, u]\left[w^{-1}, u\right] \text {, }
$$

$$
\left[u, w, u^{-1}\right]=[w, u]\left[w, u^{-1}\right] \text {. }
$$

In what follows $z=[x, y]$.

\section{Proofs of the Theorems}

Let

$$
\begin{aligned}
z & =\left[u, w, w^{-1}\right] \\
& =[w, u]\left[w^{-1}, u\right]
\end{aligned}
$$

by (2.4). Take the inverse to get

$$
\begin{aligned}
z^{-1} & =\left[u, w^{-1}\right][u, w] \\
& =\left[w^{-1}, u, w\right]
\end{aligned}
$$

by (2.5). Interchanging $x$ and $y$, we have

$$
z=\left[u^{-1}, w, u\right] \text {. }
$$

Hence a law of the form $z=\left[u, w, w^{-1}\right]$ implies a law of the form $z=\left[u^{-1}, w, u\right]$. For example

$$
z=\left[y, x, x^{-1}\right] \text { implies } z=\left[y^{-1}, x, y\right] \text {. }
$$

By the same method we can prove the converse, i.e., a law $z=\left[w^{-1}, u, w\right]$ implies the law $z=\left[w, u, u^{-1}\right]$. Hence out of sixteen cases of the first category we have to discuss only eight cases of the form $\left[u, w, w^{-l}\right]$. Numerous proofs can be given for these eight cases. We outline the method by considering a few examples.

Let

$$
\begin{aligned}
z & =\left[y, x, x^{-1}\right] \\
& \left.=[x, y]\left[x^{-1}, y\right] \quad \text { (use }(2.4)\right) .
\end{aligned}
$$


Hence $\left[x^{-1}, y\right]=1$ or $[x, y]=1$.

Similarly we can prove

$$
z=\left[y, x^{-1}, x\right] \text { implies } z=1 \text {. }
$$

Let

$$
z=\left[y^{-1}, x, x^{-1}\right] \text {. }
$$

Then

$$
z=\left[x, y^{-1}\right]\left[x^{-1}, y\right]
$$

Fut $y^{-1}$ for $y$ and solve for $[x, y]$ to get

$$
[x, y]=\left[x, y^{-1}\right]\left[y, x^{-1}\right] \text {. }
$$

From (3.4) and (3.5) we have

$$
[x, y]=\left[y^{-1}, x\right] \text {. }
$$

In (3.4) put $x^{-1}=x$ and $y^{-1}=y$ and solve for $[x, y]$ to get

$$
[x, y]=\left[y, x^{-1}\right]\left[x^{-1}, y^{-1}\right] \text {. }
$$

Take the inverses of $(3.4),(3.5)$ and (3.7) and interchange $x$ and $y$ to get

$$
\begin{aligned}
& z=\left[x^{-1}, y^{-1}\right]\left[x^{-1}, y\right] ; \\
& z=\left[y^{-1}, x\right]\left[x^{-1}, y\right] ; \\
& z=\left[x^{-1}, y^{-1}\right]\left[y^{-1}, x\right] .
\end{aligned}
$$

$(3.6)$ and $(3.10)$ gives us

$$
\left[x^{-1}, y^{-1}\right]=1 \text { or }[x, y]=1 \text {. }
$$

We also note that (3.8) and (3.9) implies

$$
[x, y]=\left[y, x^{-1}\right]
$$

while (3.6) and (3.11) imply

$$
[x, y]=\left[x^{-1}, y^{-1}\right]
$$


Using (3.11) and (3.12), we find that (3.3) also implies

$$
z=\left[x^{-1}, y^{-1}, y\right]
$$

and

$$
z=\left[y, x^{-1}, x\right]
$$

and a large number of these equivalences can be obtained. Using the above method a large number of proofs can be given for the cases of the first category .

Let us then discuss the cases for category 2 .

Let

$$
z=[y, x, x]
$$

changing $x$ into $x^{-1}$ and conjugating by $x$, we get

$$
\left[x^{-1}, y\right]^{x}=\left[y, x^{-1}, x^{-1}\right]^{x}
$$

or

$$
[y, x]=\left[x,\left[y, x^{-1}\right]\right] .
$$

Taking the inverse we get

$$
[x, y]=\left[y, x^{-1}, x\right]
$$

Hence

$$
z=[y, x, x] \text { implies } z=\left[y, x^{-1}, x\right] \text {. }
$$

Similarly we can prove

$$
\begin{gathered}
z=[x, y, x] \text { implies } z=\left[x^{-1}, y, x\right] ; \\
z=\left[x, y^{-1}, x\right] \text { implies } z=\left[x^{-1}, y^{-1}, x\right] ; \\
z=\left[y^{-1}, x, x\right] \text { implies } z=\left[y^{-1}, x^{-1}, x\right] .
\end{gathered}
$$

Now let us consider

$$
z=[y, x, y]
$$

or 


$$
\left[x, y^{-1}\right]^{-y}=\left[y^{-1}, x, y^{-1}\right]^{-y}
$$

i.e.

$$
[x, y]=\left[y^{-1}, x, y\right]
$$

Hence

$$
z=[y, x, y] \text { implies } z=\left[y^{-1}, x, y\right] \text {. }
$$

Similarly we can prove that

$$
\begin{aligned}
& z=\left[x^{-1}, y, y\right] \text { implies } z=\left[x^{-1}, y^{-1}, y\right] ; \\
& z=\left[y, x^{-1}, y\right] \text { implies } z=\left[y^{-1}, x^{-1}, y\right] ; \\
& z=[x, y, y] \quad \text { implies } z=\left[x, y^{-1}, y\right] .
\end{aligned}
$$

Since the derived laws in (3.13) to (3.20) belong to category 1 , in all these cases the original laws imply that the group is abelian.

Before discussing the last category, we shall illustrate how some of the laws discussed before could have also been proved by the following method. Let

$$
\begin{aligned}
z=[x, y] & =[x, y, x] \\
& =[z, x]=[z, x, z]=[z, z]=1 .
\end{aligned}
$$

Let

$$
\begin{gathered}
z=[y, x, y] ; \\
z=\left[z^{-1}, y\right]=\left[z^{-1}, y, z^{-1}\right]^{-1} \\
=\left[z, z^{-1}\right]^{-1}=1 .
\end{gathered}
$$

4.

As mentioned before we have to supply separate proofs for separate cases for the last category.

Let

$$
z=\left[x^{-1}, y^{-1}, x^{-1}\right]
$$

Expanding and rearranging we get 
(4.2)

$$
z=y x\left[y, x^{-1}\right] y^{-1} x^{-1}
$$

Take the inverse of (4.2) and interchange $x$ and $y$ to get

$$
z=y x\left[y^{-1}, x\right] y^{-1} x^{-1} \text {. }
$$

$(4.2)$ and $(4.3)$ give

$$
[x, y]=\left[x^{-1}, y^{-1}\right]
$$

(4.1) and (4.4) imply

$$
[x, y]=[x, y, x] \text {. }
$$

Now (4.5) belongs to the second category and hence (4.1) implies $z=1$.

In the same way we can prove that

$$
z=\left[y^{-1}, x^{-1}, y^{-1}\right] \text { implies }[x, y]=1 \text {. }
$$

Let

$$
z=\left[y^{-1}, x^{-1}, x^{-1}\right]
$$

Then

$$
z=\left[x^{-1}, y^{-1}\right]\left[y^{-1}, x^{-1}\right]^{x^{-1}} \text {. }
$$

Take the inverse and interchange $x$ and $y$ to get

$$
z=\left[y^{-1}, x^{-1}\right]^{y^{-1}}\left[x^{-1}, y^{-1}\right]
$$

In (4.8) change $x$ into $x^{-1}$ and $y$ into $y^{-1}$ and solve for $z$ to get

$$
z=\left[x^{-1}, y^{-1}\right][x, y]^{x} \text {. }
$$

(4.8) and (4.10) give

$$
\left[y^{-1}, x^{-1}\right]^{x^{-1}}=[x, y]^{x}
$$

or

$$
\begin{aligned}
{[x, y]^{x^{2}} } & =\left[y^{-1}, x^{-1}\right] \\
& =[x, y, y]
\end{aligned}
$$


from (4.7).

Gupta [1] proved that (4.11) implies an abelian group. Hence (4.7) implies that the group is abelian. Before proceeding we shall prove Theorem 2. Let

$$
[x, y]^{x^{2}}=[x, y, x]
$$

Hence

$$
z\left[z, x^{2}\right]=[z, x] .
$$

From (4.12) we get

$$
\begin{aligned}
{[z, x]^{z^{2}} } & =[z, x, z] & & \\
& =\left[z\left[z, x^{2}\right], z\right] & & (\text { use }(4.13)) \\
& =\left[z, x^{2}, z\right] & & (\text { use }(2.3)) \\
& =\left[z, x^{2}\right]^{z^{2}} & & \text { (use }(4.12)) .
\end{aligned}
$$

Therefore

$$
[z, x]=\left[z, x^{2}\right]
$$

and $z=1$ follows from $(4.13)$.

Consider

$$
[x, y]=\left[x^{-1}, y^{-1}, y^{-1}\right]
$$

or

$$
[x, y]^{-y}=\left[x^{-1}, y^{-1}, y^{-1}\right]^{-y}=\left[x^{-1}, y^{-1}, y\right] .
$$

We deduce that

$$
[y, x]^{-y}=\left[y^{-1}, x^{-1}\right]\left[y, x^{-1}\right] \quad \text { (use (2.4)). }
$$

Taking the inverse we have

$$
[x, y]^{y}=\left[x^{-1}, y\right]\left[x^{-1}, y^{-1}\right] \text {. }
$$

Now (4.14) can be written as

$$
[x, y]=\left[y^{-1}, x^{-1}\right]\left[x^{-1}, y^{-1}\right]^{y^{-1}} \text {. }
$$

Change $x$ into $x^{-1}, y$ into $y^{-1}$ and solve for $[x, y]^{y}$ to get 
Group laws

343

$(4.16)$

$$
[x, y]=[x, y]\left[x^{-1}, y^{-1}\right] \text {. }
$$

From (4.15) and (4.16) we have

$$
[x, y]=\left[x^{-1}, y\right] \text {. }
$$

Hence for (4.14) we have

$$
\left[x^{-1}, y\right]=\left[x, y^{-1}, y^{-1}\right]
$$

or

$(4.17)$

$$
[x, y]=\left[x, y^{-1}, y^{-1}\right] \text {, }
$$

i.e., a law $z=\left[x^{-1}, y^{-1}, y^{-1}\right]$ implies a law $z=\left[x, y^{-1}, y^{-1}\right]$. But since $z=\left[x, y^{-1}, y^{-1}\right]$ implies abelianness (see [1]) therefore (4.14) implies abelianness.

Let

$$
z=\left[y^{-1}, x, y^{-1}\right] \text {, }
$$

ie.,

$$
z=\left[x, y^{-1}\right]\left[y^{-1}, x\right]^{y^{-1}}
$$

Take the inverse of (4.19) and interchange $x$ and $y$ to get

$$
z=\left[y, x^{-1}\right]^{x^{-1}}\left[x^{-1}, y\right] \text {. }
$$

In (4.19) change $y$ into $y^{-1}$ and solve for $z$.

$$
\text { (4.21) }
$$

$$
z=\left[x, y^{-1}\right][x, y]^{y} \text {. }
$$

From (4.19) and (4.21) we have

$$
[x, y]^{y}=\left[y^{-1}, x\right]^{y^{-1}} \text {. }
$$

Thus

$$
z=\left[y^{-1}, x\right]^{y^{-2}}
$$

and

https://doi.org/10.1017/S0004972700042027 Published online by Cambridge University Press 


$$
\left[y^{-1}, x, y^{-1}\right]=\left[y^{-1}, x\right]^{y^{-2}} \quad(\text { use }(4.18)) \text {. }
$$

Put $y^{-1}=x$ and $x=y$ to get

$$
\text { (4.22) }[x, y]^{x^{2}}=[x, y, x] \text {. }
$$

Using Theorem 2, the required result follows.

Lastly let us consider

$$
z=\left[y, x^{-1}, x^{-1}\right]
$$

or

$$
z=\left[x^{-1}, y\right]\left[y, x^{-1}\right]^{x^{-1}}
$$

In (4.24) change $x$ into $x^{-1}$ and solve for $[x, y]$

$$
z=\left[x^{-1}, y\right][x, y]^{x}
$$

(4.24) and (4.25) give

$$
\left[y, x^{-1}\right]^{x^{-1}}=[x, y]^{x}
$$

Take the inverse and interchange $x$ and $y$ to get

$$
[x, y]^{y^{2}}=\left[y^{-1}, x\right] \text {, }
$$

or, using (4.23), we get

$$
\text { (4.27) }[x, y]^{y^{2}}=[x, y, y] \text {. }
$$

But (4.27) implies that the group is abelian (see [1]), hence the required result follows.

\section{References}

[1] N.D. Gupta, "Some group-laws equivalent to the commutative law", Arch. Math. 17 (1966), 97-102. 
[?] Marshall Hall, Jr, The theory of groups (Macmillan, New York, 1959).

York University,

Toronto, Canada,

and

Western IIIinois University,

Macomb, Illinois, USA. 